\title{
Early postoperative respiratory complications following elective craniotomies
}

Postoperative pulmonary complications (PPCs), frequently seen in the early postoperative period in neurosurgical population with an incidence of about $23 \%$, manifest mainly as pneumonia, bronchitis, atelectasis and respiratory failure. ${ }^{[1]}$ An understanding of the risk factors leading to PPCs in neurosurgical population may be helpful in reducing the morbidity and mortality.

In the heterogeneous surgical population undergoing non cardiothoracic surgery, an attempt was made, by the Clinical Efficacy Assessment Subcommittee of the American College of Physicians (ACP), for stratification of factors responsible for PPCs. ${ }^{[2]}$ Advanced age (age $>60$ years), American Society of Anesthesiologists (ASA) class of II or greater, functionally dependent patients, pre-existing chronic obstructive pulmonary disease and congestive heart failure are associated with increased risk of PPCs. Similarly prolonged surgery ( $>3$ hours), abdominal surgery, thoracic surgery, neurosurgery, head and neck surgery, vascular surgery, aortic aneurysm repair, emergency surgery, and general anaesthesia are the procedure related risk factors associated with PPCs. A low serum albumin level $(<35 \mathrm{~g} / \mathrm{L})$ is a powerful marker of increased risk for PPCs. Fair evidence supports the blood urea nitrogen levels of greater than $21 \mathrm{mg} / \mathrm{dl}$ as a predictor for increased PPCs. ${ }^{[2]}$

Pulmonary complications in neurosurgical population have not been studied extensively. Only a few authors have focussed on this issue. Although neurosurgery is a strong risk factor for development of PPCs (pooled estimated odds ratio 2.53), ${ }^{[2]}$ the causative factors for these complications have not been identified. In this issue of Journal of Neuroanaesthesia and Critical Care, Bharati et al., have published their study on the factors responsible for respiratory complications after elective craniotomies. The authors found that the commonest respiratory complication was purulent tracheobronchitis which was found in $9.4 \%$ of patients. They found on multivariate analysis that infratentorial surgery,

\begin{tabular}{|l|l|}
\hline \multicolumn{2}{|c|}{ Access this article online } \\
\hline Quick Response Code: & Website: \\
\hline & www.jnaccjournal.org \\
\cline { 2 - 2 } & \\
\hline
\end{tabular}

mechanical ventilation $>48$ hours, $>3$ days stay in the ICU, decreased level of consciousness in the post-operative period, >300 minutes of surgical duration, chronic lung diseases, hypokalaemia, fever in the post operative period and intraoperative tachycardia, were significant risk factors for postoperative respiratory complications (PRCs). The present study has tried to study the respiratory complications in the postoperative period while as previous studies mainly focussed on only PPCs.

Chu and Dang ${ }^{[3]}$ found that in patients undergoing elective craniotomy for brainstem tumours, the incidence of PPCs was $23.9 \%$. They observed that partial tumour resection is a risk factor for occurrence of PPCs as the residual tumour tissue could affect cerebral functions. Dube et al., ${ }^{[4]}$ observed that the incidence of reintubation within 24-72 hours after elective craniotomy was about $4.9 \%$. The common causes of reintubation were neurological deterioration and residual tumour with surrounding oedema. A good mentation is helpful for airway protection but copious oropharyngeal secretions could lead to reintubation even in the presence of a good cough reflex. In infratentorial surgery the cough reflex may not be well-preserved due to lower cranial nerve palsy and pharyngeal muscles dysfunction, increasing the frequency of reintubation.

In a heterogenous population of non-emergent craniotomies, Sogame et al., ${ }^{[1]}$ observed, that PPCs occurred in $24.6 \%$, and $10 \%$ of them died. On multivariate analysis, predictors for occurrence of PPCS were infratentorial surgery, mechanical ventilation $\geq 48$ hours, time spent in the ICU $>3$ days, decrease in level of consciousness, duration of surgery $\geq 300$ minutes, and pre-existing chronic lung disease. The most frequent postoperative pulmonary complication was purulent tracheobronchitis, followed by pneumonia, bronchospasm, and atelectasis. Direct brain injury from trauma, depressed level of consciousness, inability to protect the airway, disruption of natural defence barriers, decreased mobility, and secondary physiopathologic insults inherent to severe brain injury lead to the development of pulmonary complications. ${ }^{[5]}$

Pneumonia is a common PPC in neurosurgical population. Endotracheal intubation and duration of ventilation increase the incidence of pneumonia. A clinical pulmonary infection score (CPIS) is helpful in diagnosis of suspected ventilator associated pneumonia (VAP). It takes into consideration the clinical, radiographic, 
physiological (PaO2/FiO2), and microbiologic data and translates it into a single numerical result. A CPIS $>6$ has a good correlation with the presence of pneumonia when correlated with quantitative cultures of bronchoscopic, nonbronchoscopic and broncho-alveolar lavage specimens. ${ }^{[6]}$ When the CPIS $\leq 6$ a strong suspicion of VAP is made and a re-evaluation should be done after 48-72 hours.

Neurogenic pulmonary edema (NPE) is associated with acute onset of pulmonary oedema resulting from central nervous system (CNS) injury due to subarachnoid haemorrhage, severe head injury, ${ }^{[7]}$ and status epilepticus. Studies have shown that of NPE may result from higher intracranial pressures (ICP) and low cerebral perfusion pressures. ${ }^{[8]}$ The absence of a clear lung injury and a normal chest X-ray (CXR) in these patients also suggests that brain injury to be a risk factor for this phenomenon. The 'blast injury' phenomenon may explain a surge in adrenergic response leading to an increase in capillary pressures in the lung bed, and endothelial damage. This leads to a capillary leak into the alveoli and pulmonary interstitium ${ }^{[9]}$ associated with production of inflammatory mediators such as IL-6. ${ }^{[10,11]}$ A 'double hit' model has been proposed in patients with a brain injury. The 'first hit' may be the result of a combination of an adrenergic surge and systemic production of inflammatory mediators, making the lung more susceptible to injury, and a 'second hit' may be from extracorporeal variables such as infections, transfusions, and mechanical ventilation. ${ }^{[12]}$

Lung atelectasis occurs in neurosurgical population due to respiratory depression, and impaired consciousness leading to aspiration. ${ }^{[13]}$ Weakness of thoracoabdominal muscles due to cervical and thoracic spine injury may lead to hypoventilation and bronchial obstruction from mucous plugs. Associated injuries as traumatic pneumothorax, hemothorax may be contributing factors.

Pulmonary thromboembolism is common in neurosurgical population due to chronic immobility. It manifests as acute onset dyspnoea, hypoxemia, or hypotension. Initial screening by D-dimer, lower limb compression ultrasound or multidetector CT (MDCT) angiography are helpful. Neurosurgical patients should receive thromboprophylaxis by mechanical or pharmacological means. It has been seen that the majority of deep venous thrombi (DVTs) occurred within the first week after a neurosurgical procedure, with a linear correlation between the duration of surgery and DVT occurrence. ${ }^{[14]}$ Use of early subcutaneous heparin (at either 24 or 48 hours) was associated with a $43 \%$ reduction of developing a lower-extremity DVT, without an increase in surgical site haemorrhage, ${ }^{[14,15]}$ After craniotomy, low dose subcutaneous heparin (5000U BID or TID) starting after the second day significantly reduces the frequency of venous thromboembolism, with no increase in incidence of intracranial bleeding. ${ }^{[16]}$ Treatment with low molecular weight heparin (that is, enoxaparin $40 \mathrm{mg}$ daily) is a reasonable alternative if renal function is normal, and the results of recent studies suggest that both are equally effective with similar rates of heparin-induced thrombocytopenia. ${ }^{[17]}$ When contraindicated, the use of inferior vena cava filters may be necessary in the short term. ${ }^{[15]}$

Acute respiratory distress syndrome and acute lung injury are frequently seen in neurosurgical population. Recent developments in the therapeutic approach to ARDS include emphasis on protective lung ventilation using low tidal volumes, increased PEEP with use of recruitment manoeuvres to promote reopening of collapsed lung alveoli, prone position as rescue therapy for severe hypoxemia, and high frequency ventilation. Supportive measures include the attention to fluid balance, restrictive transfusion strategies, and minimization of sedatives and neuromuscular blocking agents. Inhaled bronchodilators, (nitric oxide and prostaglandins), use of corticosteroids and extracorporeal oxygenation have shown short term improvement in patients with intractable hypoxemia. ${ }^{[18]}$

Strategies for prevention of postoperative pulmonary complications following craniotomy:

The strategies which are helpful for the prevention of postoperative respiratory complications include thromboprophylaxis by mechanical and pharmacological means. Prophylactic strategies for venous thromboembolism should be instituted within the first week postoperatively. ${ }^{[14]}$ American College of Physicians guidelines, ${ }^{[2]}$ have graded evidence for strategies to reduce risk for PPCs. Postoperative lung expansion modalities (incentive spirometry, deep breathing exercises, intermittent positive-pressure breathing, and continuous positive airway pressure) are the only strategies, supported by good evidence for reduction in PPCs. Selective rather than routine use of nasogastric tubes and short-acting neuromuscular blockade decreases the risk of PPCs. ${ }^{[19]}$

Orotracheal intubation may be preferred to nasotracheal intubation for urgent intubation in neurosurgical patients as the latter may lead to an increased risk of sinusitis and pneumonia. Early tracheostomy should be considered in patients with a high cervical spinal cord injury and in those with a need for prolonged duration of mechanical ventilation. Bedside percutaneous dilatational tracheostomy has increasingly become the technique of choice for tracheostomy in neurosurgical patients. ${ }^{[20]}$ It appears to have a low incidence of complications in neurosurgical patients and shortens the hospital stay when compared with traditional surgical tracheostomy. 


\section{Areas for future research}

Most of the neurological patients have a lower Glasgow Coma Scale (GCS) on admission. During their hospital stay they have more ICU and ventilator-days, with more early tracheostomies, more VAP rates when compared with those of non-neurological patients. ${ }^{[21]}$ However studies have shown that the rate of reintubation is similar to those of non-neurological patients. Hence mental status and GCS may not be the only predictors for successful extubation, ${ }^{[22]}$ as GCS may be higher in nonneurological patients and the rate of reintubation being the same. It is not clear as to why neurological patients are more likely to get 'stuck' on the ventilator or may need early reintubation. The other factors that need to be addressed in this scenario are the age, neurological diagnosis, characteristics of tracheobronchial secretions, cranial nerve involvement, pupillary abnormalities and absence of gag reflex. ${ }^{[23]}$ These questions need to be addressed in multicentric prospective clinical trials. Meanwhile the clinical expertise of a neurointensivist may guide the best approach to a particular patient.

\section{Zulfiqar Ali}

\section{Department of Anesthesiology, Division of Neuroanesthesolgy, Sher-I-Kashmir Institute of Medical Sciences, Srinagar, Jammu and Kashmir, India Address for correspondence: Dr. Zulfiqar Ali, \\ Division of Neuroanesthesiolgy, Sher-I-Kashmir Institute of Medical Sciences, Srinagar - 190 011, Jammu and Kashmir, India E-mail: zulfiqaraliiii@yahoo.com}

\section{REFERENCES}

1. Sogame LC, Vidotto MC, Jardim JR, Faresin SM. Incidence and risk factors for postoperative pulmonary complication in elective intracranial surgery. J Neurosurg 2008;109:222-7.

2. Qaseem A, Snow V, Fitterman N, Hornbake ER, Lawrence VA, Smetana GW, et al. Clinical Efficacy Assessment Subcommittee of the American College of Physicians. Risk assessment for and strategies to reduce perioperative pulmonary complications for patients undergoing non cardiothoracic surgery: A guideline from the American College of Physicians. Ann Intern Med 2006; 144:575-80.

3. Chu H, Dang BW. Risk factors of postoperative pulmonary complications following elective craniotomy for patients with tumors of the brainstem or adjacent to the brainstem. Oncol Lett 2014;8:1477-81.

4. Dube SK, Rath GP, Bharti SJ, Bindra A, Vanamoorthy P, Gupta N, et al. Causes of tracheal re-intubation after craniotomy: A prospective study. Saudi J Anaesth 2013;4:410-4.

5. Lee K, Rincon F. Pulmonary Complications in Patients with Severe Brain Injury. Crit Care Res Pract 2012;2012:207247.

6. Pugin J, Auckenthaler R, Mili N, Janssens JP, Lew PD, Suter PM. Diagnosis of ventilator-associated pneumonia by bacteriologic analysis of bronchoscopic and nonbronchoscopic "blind" bronchoalveolar lavage fluid. Am Rev Respir Dis 1991;143:1121-9.

7. Rogers FB, Shackford SR, Trevisani GT, Davis JW, Mackersie RC, Hoyt DB. Neurogenic pulmonary edema in fatal and nonfatal head injuries. J Trauma 1995;5:860-8.

8. Touho H, Karasawa J, Shishido H, Yamada K, Yamazaki Y. Neurogenic pulmonary edema in the acute stage of hemorrhagic cerebrovascular disease. Neurosurgery 1989;5:762-8.

9. Theodore J, Robin ED. Speculations on neurogenic pulmonary edema (NPE). Am Rev Resp Dis 1976;4:405-11.

10. Ott L, McClain CJ, Gillespie M, Young B. Cytokines and metabolic dysfunction after severe head injury. J Neurotrauma 1994;5:447-72.

11. Mckeating EG, Andrews PJ, Signorini DF, Mascia L. Transcranial cytokine gradients in patients requiring intensive care after acute brain injury. Br J Anaesth 1997;5:520-3.

12. Mascia L. Acute lung injury in patients with severe brain injury: A double hit model. Neurocritical Care 2009;3:417-26.

13. Guleria R, Madan K. Pulmonary complications in neurosurgical patients. Indian J Neurosurg 2012;1:175-80.

14. Khaldi A, Helo N, Schneck MJ, Origitano TC. Venous thromboembolism: Deep venous thrombosis and pulmonary embolism in a neurosurgical population. J Neurosurg 2011;114:40-6.

15. Guyatt GH, Akl EA, Crowther M, Gutterman DD, Schuunemann HJ. Executive summary: Antithrombotic therapy and prevention of thrombosis, $9^{\text {th }}$ ed: American College of Chest Physicians Evidence-Based Clinical Practice Guidelines. Chest 2012;141:7S-47.

16. Boeer A, Voth E, Henze T, Prange HW. Early heparin therapy in patients with spontaneous intracerebral haemorrhage. J Neurol Neurosurg Psychiatry 1991;54:466-7.

17. Cook D, Meade M, Guyatt G, Walter S, Heels Ansdell D, Warkentin TE, et al. Dalteparin versus unfractionated heparin in critically ill patients. N Engl J Med 2011;364:1305-14.

18. Pierrakos C, Karanikolas M, Scolletta S, Karamouzos V, Velissaris D. Acute respiratory distress syndrome: Pathophysiology and therapeutic options. J Clin Med Res 2012;4:7-16.

19. Smetana GW. Postoperative pulmonary complications: An update on risk assessment and reduction. Cleve Clin J Med 2009;76:S60-5.

20. Browd SR, MacDonald JD. Percutaneous dilational tracheostomy in neurosurgical patients. Neurocrit Care 2005;2:268-73.

21. Pelosi P, Ferguson ND, Frutos-Vivar F, Anzueto A, Putensen C, Raymonds K, et al. Management and outcome of mechanically ventilated neurologic patients. Crit Care Med 2011;39:1482-92.

22. Coplin WM, Pierson DJ, Cooley KD, Newell DW, Rubenfeld GD. Implications of extubation delay in brain injured patients meeting standard weaning criteria. Am J Respir Crit Care Med 2000;161:1530-6.

23. Karanjia N, Nordquist D, Stevens R, Nyquist P. A clinical description of extubation failure in patients with primary brain injury. Neurocrit Care 2011;15:4-12.

How to cite this article: Ali Z. Early postoperative respiratory complications following elective craniotomies. J Neuroanaesthesiol Crit Care 2015;2:85-7.

Source of Support: Nil, Conflict of Interest: None declared. 